\title{
Salurtepe Dağı (Elmalı-Antalya) Ardıç Ormanında Güncel Polen Dağılımının İncelenmesi
}

\section{Modern Pollen Distribution of Juniperus Forest in Salurtepe Mountain (Elmall-Antalya)}

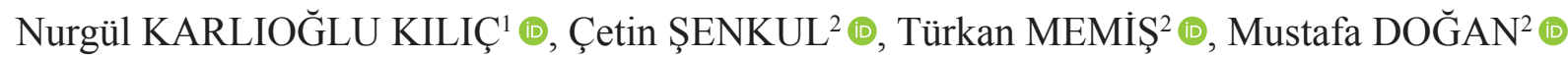

'İstanbul Üniversitesi-Cerrahpaşa, Orman Fakültesi, Orman Botaniği Anabilim Dalı, İstanbul, Türkiye

${ }^{2}$ Süleyman Demirel Üniversitesi, Fen Edebiyat Fakültesi, Coğrafya Bölümü, Isparta, Türkiye

ORCID: N.K.K. 0000-0002-6255-6819; Ç.Ş. 0000-0002-7641-1143; T.M. 0000-0002-5442-5595; M.D. 0000-0002-0124-9866

\section{öz}

Bu çalışma, Salurtepe Dağı́nda (Elmalı-Antalya) bulunan Juniperus sp. (Ardıç) Ormanı'nda bulunan bitki kompozisyonunun değişkenlik gösterdiği 4 farklı örnek alanda, 2015-2017 yılları arasında güncel odunsu ve otsu bitki polen yoğunluğunu (cm²/yıl) belirlemek amacıyla gerçekleştirilmiştir. Dört ayrı örnek alana 2015 yılında Tauber polen tuzakları yerleştirilmiş ve bu tuzaklar 2015-2017 yılları arasında yıllık olarak değiştirilmiştir. Her 1 yıllık dönem için tuzakların etrafından kara yosunu örnekleri de toplanmıştır. Hem Tauber polen tuzaklarındaki kimyasal karışım, hem de kara yosunu örnekleri “Avrupa Polen İzleme Programı Protokolü (European Pollen Monitoring Programme Protocol: EPMPP)"ne göre analiz edilmiştir. Polen tuzaklarından elde edilen polen preparatlarında 2015-2016 yılına ait odunsu bitki polen yoğunluğu değişimleri incelendiğinde Cedrus libani, Pinus sp., Juniperus sp. ve Quercus coccifera'nın polen yoğunlukları fazla iken, 2016-2017 yılında Juniperus sp., Cedrus libani, Juglans sp., Pinus sp., Quercus coccifera ve Rosaceae familyasının polen yoğunlukları fazla çıkmıştır. Kara yosunu örneklerinden elde edilen polen preparatlarında 2015-2016 ve 2016-2017 yıllarına ait odunsu bitki polen yoğunluğu değişimlerine bakıldığında Cedrus libani, Juniperus sp., Pinus sp. ve Quercus coccifera'nın polen yoğunlukları fazla çıkmıştır. Hem polen tuzakları hem de yosun örneklerine ait 2 yıllık polen yoğunluğu verileri güncel vejetasyonu yansıtmaktadır.

Anahtar kelimeler: Tauber polen tuzağı, Juniperus, polen yoğunluğu

\section{ABSTRACT}

This study was carried out to determine the modern pollen influx ( $\mathrm{cm}^{2} /$ year) of arboreal and herbaceous plants in 4 different sample areas where vegetation varied in Juniperus L. (Juniper) Forest in the Salurtepe mountain between 2015 and 2017. Tauber pollen traps were placed in four sample areas in 2015 and these traps were changed annually between 2015 and 2017. For every 1-year period, moss samples were also collected from around the traps. Both the chemical mixture in the Tauber pollen traps and the moss samples were analysed according to the "European Pollen Monitoring Programme Protocol". When the arboreal pollen influx of pollen preparations obtained from pollen traps was examined, the pollen influxes of Cedrus libani, Pinus sp., Juniperus sp. and Quercus coccifera were higher between 2015 and 2016. In 2016-2017, pollen influxes of Juniperus sp., Cedrus libani, Juglans sp., Pinus sp., Quercus coccifera and Rosaceae family increased. When the arboreal pollen influx of pollen preparations obtained from moss samples was examined, the pollen influxes of Cedrus libani, Juniperus sp., Pinus sp. and Quercus coccifera were higher in the periods of 2015-2016 and 2016-2017. Two-year pollen influx data of both pollen traps and moss samples reflects the current vegetation.

Keywords: Tauber pollen trap, Juniperus, modern pollen influx

Başvuru/Submitted: 21.02.2019 • Revizyon Talebi/Revision Requested: 08.03.2019 • Son Revizyon/Last Revision Received: 13.03 .2019 • Kabul/Accepted: 19.03.2019 • Online Yayın/Published Online: 15.04.2019 


\section{EXTENDED ABSTRACT}

This study was carried out to determine the modern pollen influx ( $\mathrm{cm}^{2} /$ year) of arboreal and herbaceous plants in 4 different sample areas where vegetation varied in Juniperus L. (Juniper) Forest in the Salurtepe mountain (Elmal1-Antalya) between 2015 and 2017. Tauber pollen traps were placed in four sample areas in 2015 and these traps were changed annually between 2015 and 2017 . For every 1-year period, moss samples were also collected from around the traps. The modern plants around the traps (0-10.5 m) were identified using the vegetation ring. In addition, a surface sediment sample was taken from Elmalı Lake in the southeast of the study area and the modern pollen distribution of the plants around the lake was also revealed. Both the chemical mixture in the Tauber pollen traps and the moss samples were analysed according to the "European Pollen Monitoring Programme Protocol (EPMPP)".

According to this protocol, the mixture in each Tauber pollen trap taken from the field was filtered through a $250 \mathrm{~m} \mu$ pollen sieve and removed from animal and plant residues. Then 3 Lycopodium spore tablets $(10 \mathrm{ml}$ distilled water and $0.5 \mathrm{ml} \mathrm{HCl}$ solution) were added to this mixture. The resulting chemical mixture was transferred to centrifuge tubes and centrifuged until it reached sediment (10 minutes at 3000-3500 rpm). Then $10 \mathrm{ml}$ of acetolysis mixture (9 volumes of acetic anhydride and 1 volume of sulfuric acid) was added to the resulting sediment. After the acetolysis step, $2 \mathrm{ml}$ glycerin was added to prepare pollen preparations. Counting and diagnosis of the pollen grains and Lycopodium spores was made by using computer-assisted Leica DM750 brand light microscope, x40, x100 immersion lens and 10x ocular.

As a result of the field studies, 4 moss samples were collected and analyzed by following the principles of EPMPP. According to this protocol, Potassium Hydroxide (KOH 10\%) was added to the moss samples respectively and the samples were stored in a hot water bath. The moss samples removed from the water bath were filtered through a 180 mesh sieve and the Lycopodium spore tablet was added to the filtered sample. Pollen fluid belonging to the moss samples was reduced to a single tube by centrifugation ( 5 minutes at $4000 \mathrm{rpm}$ ). The same procedures of counting pollen grains and Lycopodium spores were applied in the pollen slides of moss samples. In addition, with the Glew Corer sampling from Elmalı Lake, a surface sediment sample was collected in 2017 and the first $2 \mathrm{~cm}$ of this sample was analyzed according to the classical fossil pollen method.

When the arboreal pollen influx (AP) of pollen preparations obtained from pollen traps was examined, the pollen influxes of Cedrus libani, Pinus sp., Juniperus sp. and Quercus coccifera were higher between 2015 and 2016. In 2016-2017, pollen influxes of Juniperus sp., Cedrus libani, Juglans sp., Pinus sp., Quercus coccifera and Rosaceae family increased. When the herbaceous pollen influxes (NAP) were investigated, the pollen influx of Euphorbia sp. and Asteraceae were higher in the period of 2015-2016. And, it was determined that the pollen influxes of Poaceae, Apiaceae, Asteraceae and Euphorbia sp. increased between 2016 and 2017.

When the arboreal pollen influx (AP) of pollen preparations obtained from moss samples was examined, the pollen influxes of Cedrus libani, Juniperus sp., Pinus sp. and Quercus coccifera were higher in the periods of 2015-2016 and 2016-2017. The annual arboreal plant pollen influx of the surface sediment sample taken from Elmalı Lake near the study area is $10229 \mathrm{~cm}^{2} / \mathrm{year}$. Pinus sp. (9601 $\mathrm{cm}^{2} /$ year) comes first in this pollen influx and followed by Cedrus libani $\left(329 \mathrm{~cm}^{2} /\right.$ year). Other important arboreal pollen influxes belong to Olea europea, Quercus cerris type and Quercus ilex type. The herbaceous taxa with the highest pollen influxes are Lactucaceae (239 $\mathrm{cm}^{2} /$ year), Asteraceae and Chenopodiaceae $\left(90 \mathrm{~cm}^{2} /\right.$ year).

The total pollen influx obtained from the traps in 2015-2017 was higher than the total pollen influx obtained from the moss samples in the all sample areas. According to these results of pollen analysis in pollen traps and moss samples between 2015-2017, the annual pollen influxes ( $\mathrm{cm}^{2} /$ year) of Cedrus libani, Pinus sp., Juniperus, Quercus coccifera, Rosaceae, Juglans sp., Pinus sp., Euphorbia, Asteraceae, Poaceae, Apiaceae, Rosaceae, Lamiaceae, Caryophyllaceae, Brassicaceae and Chenopodiaceae were higher. 2-year pollen influx data of both pollen traps and moss samples reflect the modern vegetation in the Juniperus forest. Pinus sp. which has a high pollen influx in the arboreal taxa, does not exist in the vegetation ring, shows that pollen grains of Pinus come from the field with very long distances. 


\section{GİRIŞ}

Kuvaterner çalışmalarında fosil polen araştırmalarının daha doğru yorumlanabilmesi için değişik coğrafyalarda güncel polen izleme çalışmalarının gerekliliğinin anlaşılması fikri ile standart bir metot geliştirilmiştir. Bu amaç doğrultusunda, 1996 yılında Avrupa Polen İzleme Programı Protokolü (European Pollen Monitoring Programme Protocol: EPMPP) oluşturulmuştur (Giesecke vd., 2010). Bu protokol sayesinde Avrupa'nın birçok ülkesinde farklı vejetasyon alanlarında Tauber polen tuzaklarının (Tauber, 1974) yerleştirildiği güncel polen izleme istasyonları kurulmuştur (Hicks, 1985; Hicks ve Hyvärinen, 1986; Kvavadze, 1999; Tonkov vd., 2001; Gerasimidis vd., 2006; Pidek, 2007; van der Knaap vd., 2001; Filipova-Marinova vd., 2010). Türkiye'de ise bu protokole uygun ilk güncel polen izleme çalışması Karlıoğlu (2011) tarafından 2007 yılında Istranca ve Belgrad Ormanları'nda gerçekleştirilmiş ve 2 yıl boyunca aylık güncel polen yoğunluğu verileri 12 farklı noktada izlenmiştir. Bu çalışmadan sonra bu konudaki araştırmaların sayısı gittikçe artmıştır (Karlıoğlu ve Akkemik, 2012; Karlıoğlu vd., 2014; Karlığlu vd., 2015; Doğan, 2017; Şenkul ve Doğan, 2018; Şenkul vd., 2018a; 2018b; 2018c). Ancak Türkiye özelinde Güneybatı Anadolu'da şimdiye kadar gerçekleştirilen fosil polen analizlerine dayalı araştırmalarda güncel polen özelliklerinin kullanımı çok düşük düzeyde kalmıştır (van Zeist vd., 1975; Bottema ve Woldring, 1984; Eastwood, 1997; Vermoere vd., 2002; Kaniewski vd., 2007; Bakker vd., 2011). Bu kapsamda
Teke Yarımadası'nda genellikle paleovejetasyon ve paleoiklimle ilgili çalışmalar (van Zeist vd., 1975; Bottema ve Woldring, 1984; Eastwood, 1997) yapılmış olmasına rağmen, sahanın bitki kompozisyonuna ait güncel polen yoğunluğu ile ilgili araştırmaların sayısı oldukça azdır (Şenkul vd., 2018c).

Bu eksikliği gidermek için Salurtepe Dağı'nda bulunan Juniperus sp. (Ardıç) ormanında vejetasyonun farklılık gösterdiği 4 örnek alanda Avrupa Polen İzleme Programı Protokolü'ne uygun Tauber polen tuzakları araziye yerleştirilmiştir. Çalışmanın amacı; polen tuzakları ve kara yosunu örneklerinde güncel polen analizleri yaparak bu ormanlardaki odunsu ve otsu taksonların güncel polen yoğunluğunu belirlemektir. Bu çalışma Güneybatı Anadolu'da daha önce yapılan fosil polen diyagramlarının ve ileride yapılacak olan paleovejetasyon, paleoiklim ve paleoekolojik değişimlerin niceliksel rekonstrüksiyonlarının daha doğru yorumlanabilmesi için temel bir kalibrasyon şeması olacaktır.

\section{VERİ VE YÖNTEM}

\section{1. Çalışma Alanı}

Çalışma alanı Türkiye'nin güney batısında, Teke Yarımadası'nda, Gölhisar ve Elmalı ilçe merkezleri arasında yer alan Salurtepe Dağı'nın üzerinde doğu-batı doğrultusunda uzanan alanı kapsamaktadır (Şekil 1). Çalışma alanı ve yakın

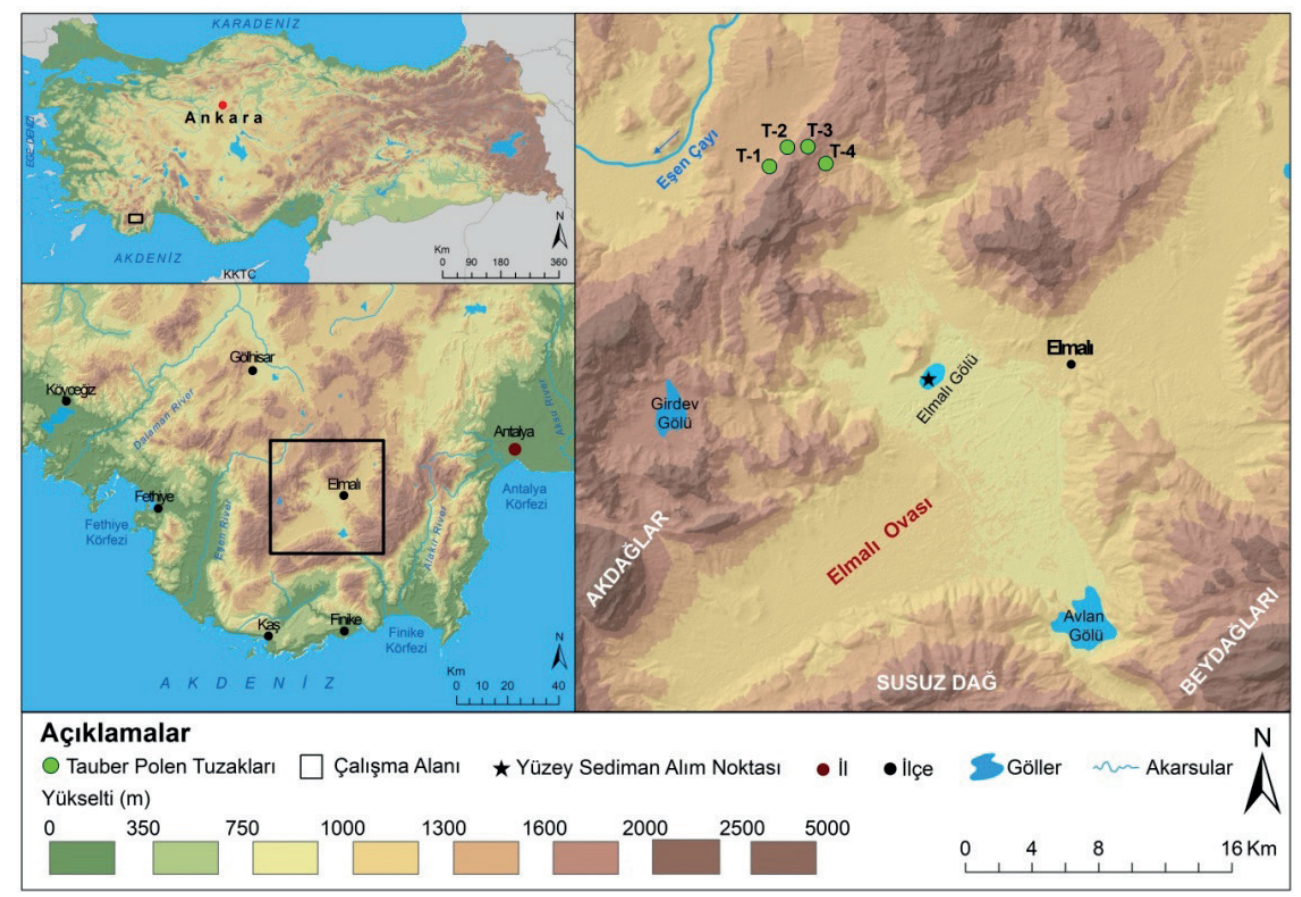

Şekil 1: Çalışma alanı lokasyon haritası.

Figure 1: The location map of the study area. 
Tablo 1: Elmalı Meteoroloji İstasyonuna ait sıcaklık, nem ve yağış ortalamaları (Meteoroloji Genel Müdürlüğü).

Table 1: Temperature, humidity and precipitation averages of the Elmali Meteorology Station (Turkish State Meteorological Service).

\begin{tabular}{|c|c|c|c|c|c|c|c|c|c|c|c|c|c|}
\hline Elmalı (1958-2015) & 0 & Ş & $M$ & $\mathbf{N}$ & $M$ & H & $\mathbf{T}$ & A & $\mathbf{E}$ & $\mathbf{E}$ & $\mathbf{K}$ & A & Yil Ort. \\
\hline$\overline{\left.\text { Ort. SIcaklık ( }{ }^{\circ} \mathrm{C}\right)}$ & 2.4 & 3.3 & 6.9 & 11.3 & 16.1 & 20.9 & 24.3 & 24.1 & 19.9 & 14.2 & 8.6 & 4.1 & 12.9 \\
\hline Ort. Nem (\%) & 71 & 67.5 & 60.5 & 54.6 & 52.2 & 44.8 & 39.2 & 39.9 & 44.6 & 55.1 & 64 & 71.9 & 55.4 \\
\hline Top. Yağ. Ort. (mm) & 82.3 & 59.4 & 47.6 & 32.1 & 28 & 21.2 & 10.1 & 8 & 7.6 & 32.9 & 46.6 & 85.5 & 461.3 \\
\hline
\end{tabular}

çevresinde bitki örtüsünün dağılışını ve karakterini belirleyen parametrelerin başında gelen faktör iklim koşullarıdır. Bu alana en yakın meteoroloji istasyonu Elmalı ilçe merkezinde bulunmaktadır. Elmalı Meteoroloji İstasyonu (1958-2015) verilerine göre; yıllık ortalama yağış $461.3 \mathrm{~mm}$, ortalama sıcaklık $12.9^{\circ} \mathrm{C}$ 'dir (Tablo 1). Çalışma alanı ve yakın çevresinde yükselti, bakı, denize olan uzaklık ve dağların uzanış yönleri, sıcaklık, yağış, rüzgâr gibi klimatik faktörler kısa mesafeler içerisinde değişmektedir.

\subsection{Polen Analizleri}

Araziden Palinoloji Laboratuvarı'na getirilen her bir Tauber polen tuzağındaki (Tauber, 1974) karışım, Avrupa Polen İzleme Programı Protokolü'ne göre $250 \mathrm{~m} \mu$ 'luk polen eleğinden süzülmüş, hayvan ve bitki kalıntılarından uzaklaştırılmıştır (Hicks vd., 1996). Daha sonra bu karışıma 3 adet Lycopodium spor tablet (Stockmarr, 1971), $10 \mathrm{ml}$ destile su ve $0.5 \mathrm{ml} \mathrm{HCl}$ 'den oluşan çözelti eklenmiştir. Her bir spor tablet 9666 adet Lycopodium sporu içermektedir. Bilinen sayıda sporun karışıma eklenmesi, preparat yapımı sırasında polen kayıplarının hesaplanmasında ve birim alana düşen polen yoğunluğunun ( $\mathrm{cm}^{2} / \mathrm{y} 11$ ) bulunmasında kullanılmaktadır (Hicks vd., 1996; Hicks vd., 2001; Tonkov vd., 2001). Elde edilen kimyasal karışım santrifüj tüplerine aktarılarak, sedimana ulaşana kadar (30003500 devirde 10 dakika) santrifüj yapılmıştır. Daha sonra $10 \mathrm{ml}$ asetoliz karışımı (9 hacim Asetik anhidrit ve 1 hacim Sülfürik asit) elde edilen sedimana eklenmiştir. Asetoliz aşamasından sonra $2 \mathrm{ml}$ gliserin eklenerek polen preparatları hazırlanmış ve her preparatın üstüne polen toplama tarihleri ile örnek alanı yazılmıştır. Polen preparatlarındaki polen ve Lycopodium'ların sayım ve teşhisi bilgisayar destekli Leica DM750 marka 1şık mikroskobunda, x40, x100 immersiyon objektifi ve 10x oküleri kullanılarak yapılmıştır. Polen teşhisleri için Palinoloji Laboratuvarındaki referans polen preparatları ile birlikte polen atlasları kullanılmıştır. (Wodehouse, 1935; Erdtman, 1952; Erdtman, 1957; Faegri ve Iversen, 1964; Aytuğ, 1967; Aytuğ vd., 1971; Iwanami vd., 1988; Moore vd., 1991; Hesse vd., 2009).

Arazi çalışmaları sonucunda polen tuzaklarının yakın çevresinden toplam 4 adet kara yosunu örneği alınmış ve bu kara yosunu örneklerine uygulanan laboratuvar metodolojisinde yine EPMPP'nin esasları takip edilmiştir. Takip edilen protokole göre sırasıyla kara yosunu örneklerinin üzerine Potasyum Hidroksit (KOH \%10'luk) eklenmiş ve örnekler sıcak su banyosunda bekletilmiştir. Su banyosundan çıkartılan kara yosunu örnekleri $180 \mathrm{~m} \mu$ 'luk elekten süzülmüş ve süzülen örneğin üzerine eritilmiş Lycopodium spor tablet eklenmiştir. Kara yosunu örneklerine ait polenli sıvı santrifüj işlemi (4000 devirde 5 dakika) ile tek tüpe düşürülmüsstür. Tek tüpe düşen örneğe Tauber polen tuzaklarının analizinde (tek tüpe düştükten sonra uygulanan işlemler), sayımında ve diyagramların elde edilmesinde uygulanan işlemler uygulanmıştır.

Ayrıca Elmalı Gölü’nden Glew Corer örnek alıcısı (Glew, 1995) ile 2017 yılında yüzey sediman örneği alınmış ve bu örneğin ilk 2 cm'lik kısmı klasik fosil polen yöntemine (Faegri ve Iversen, 1975; Moore vd., 1991) göre analiz edilmiştir.

\section{BULGULAR}

Salurtepe Dağı-Elmalı Juniperus (J) ormanından elde edilen bulgular; polen tuzaklarının çevresindeki bitki türleri ile Tauber polen tuzakları, kara yosunu örnekleri ve yüzey sediman örneğine ait polen yoğunluğu verilerinden oluşmaktadır.

\subsection{Polen Tuzaklarının Çevresindeki Bitki Türleri}

Çalışma alanı içerisinde tüm örnek alanlardaki (J-1-B, J-2-B, J-3-B ve J-4-B) polen tuzaklarının çevresindeki bitki türlerinin belirlenmesi, polenlerin ne kadarlık bir mesafeden taşındığının tespiti açısından önemlidir. Bu nedenle her bir örnek alana yerleştirilen polen tuzağının çevresinde 1'er m aralıklarla 0.5 m'den başlayarak 10.5 m'ye kadar belirlenen alanlarda odunsu ve otsu bitki taksonları tespit edilmiştir (Tablo 2).

J-1-B örnek alanı içerisinde yer alan polen tuzağı çevresindeki odunsu bitkileri Juniperus excelsa M. Bieb. ve Berberis crataegina DC. temsil etmektedir. Her iki odunsu tür de polen tuzağından 7.5 m'den itibaren bulunmaktadır. Polen tuzağına en yakın bulunan otsu bitki taksonu Euphorbia sp.'dir. J-2-B ve J-3-B örnek alanlarında yer alan polen tuzaklarının etrafında, J-1-B'deki aynı 
Tablo 2: Salurtepe Dağı Juniperus (J) ormanında polen tuzakları çevresindeki bitki taksonları.

Table 2: Plant taxa around the pollen traps in the Juniperus (J) Forest of Salurtepe Mountain

\begin{tabular}{|c|c|c|c|c|}
\hline $\begin{array}{c}\text { Polen Tuzağına } \\
\text { Uzaklık (m) }\end{array}$ & $J-1-B$ & J-2-B & J-3-B & J-4-B \\
\hline $0-0.5 \mathrm{~m}$ & & & Alkanna areolata, Cicerbita sp. & $\begin{array}{l}\text { Cicerbita sp., } \\
\text { Aubrieta pinardii, } \\
\text { Alkanna areolata. }\end{array}$ \\
\hline $0.5-1.5 \mathrm{~m}$ & Euphorbiasp. & & $\begin{array}{l}\text { Alkanna areolata. Cicerbita sp., } \\
\text { Euphorbia sp., } \\
\text { Poa bulbosa }\end{array}$ & $\begin{array}{l}\text { Cicerbita sp., Euphorbia sp., } \\
\text { Poa bulbosa, Aubrieta pinardii, } \\
\text { Alkanna areolata. }\end{array}$ \\
\hline $1.5-2.5 \mathrm{~m}$ & $\begin{array}{l}\text { Astragalus angustifolius, } \\
\text { Astragalus sp., Euphorbia sp., } \\
\text { Geranium } \\
\text { subcaulescens }\end{array}$ & $\begin{array}{l}\text { Astragalus angustifolius } \\
\text { Astragalus sp., Euphorbia sp., } \\
\text { Geranium subcaulescens }\end{array}$ & $\begin{array}{l}\text { Alkanna areolata. Euphorbia sp., } \\
\text { Poa bulbosa }\end{array}$ & $\begin{array}{l}\text { Euphorbia sp., } \\
\text { Poa bulbosa, } \\
\text { Aubrieta pinardii, Alkanna } \\
\text { areolata. }\end{array}$ \\
\hline 2.5-3.5 m & $\begin{array}{l}\text { Astragalus } \\
\text { angustifolius, } \\
\text { Euphorbia sp., Geranium } \\
\text { subcaulescens }\end{array}$ & $\begin{array}{l}\text { Astragalus } \\
\text { angustifolius, Euphorbia sp., } \\
\text { Geranium } \\
\text { subcaulescens }\end{array}$ & $\begin{array}{l}\text { Alkanna areolata, Euphorbia sp., } \\
\text { Poa bulbosa }\end{array}$ & $\begin{array}{l}\text { Euphorbia sp., } \\
\text { Poa bulbosa, } \\
\text { Alkanna areolata. }\end{array}$ \\
\hline 3.5-4.5 m & $\begin{array}{l}\text { Astragalus angustifolius, } \\
\text { Geranium } \\
\text { subcaulescens }\end{array}$ & $\begin{array}{l}\text { Astragalus } \\
\text { angustifolius, Geranium } \\
\text { subcaulescens }\end{array}$ & $\begin{array}{l}\text { Alkanna areolata, Euphorbia sp., } \\
\text { Ajuga sp., } \\
\text { Ranunculus sp. }\end{array}$ & $\begin{array}{l}\text { Alkanna areolata, } \\
\text { Ajuga sp., } \\
\text { Ranunculus sp. }\end{array}$ \\
\hline 4.5-5.5 m & $\begin{array}{l}\text { Astragalus } \\
\text { angustifolius, Euphorbia sp., } \\
\text { Geranium } \\
\text { subcaulescens }\end{array}$ & $\begin{array}{l}\text { Astragalus } \\
\text { angustifolius, Euphorbia sp., } \\
\text { Geranium } \\
\text { subcaulescens }\end{array}$ & $\begin{array}{l}\text { Euphorbia sp., Ranunculus } \\
\text { sp., Teucrium chamaedrys L., } \\
\text { Umbilicus erectus DC. }\end{array}$ & $\begin{array}{l}\text { Euphorbia sp., Ranunculus sp., } \\
\text { Teucrium } \\
\text { chamaedrys, } \\
\text { Umbilicus erectus }\end{array}$ \\
\hline $5.5-6.5 \mathrm{~m}$ & $\begin{array}{l}\text { Astragalus } \\
\text { angustifolius, Euphorbia sp., } \\
\text { Geranium } \\
\text { subcaulescens, Taraxacum sp. }\end{array}$ & $\begin{array}{l}\text { Astragalus } \\
\text { angustifolius, Euphorbia sp., } \\
\text { Geranium } \\
\text { subcaulescens, Taraxacum sp. }\end{array}$ & $\begin{array}{l}\text { Alkanna areolata, Euphorbia } \\
\text { sp., Capsella bursa-pastoris (L.) } \\
\text { Medik. }\end{array}$ & $\begin{array}{l}\text { Euphorbia sp., Alkanna areolata, } \\
\text { Capsella bursa-pastoris }\end{array}$ \\
\hline $6.5-7.5 \mathrm{~m}$ & Muscarisp. & Muscarisp. & $\begin{array}{l}\text { Alkanna areolata, Poa bulbosa, } \\
\text { Ranunculus sp., Capsella bursa- } \\
\text { pastoris }\end{array}$ & $\begin{array}{l}\text { Euphorbia sp., } \\
\text { Poa bulbosa, Alkanna areolata, } \\
\text { Ranunculus sp., Capsella bursa- } \\
\text { pastoris }\end{array}$ \\
\hline 7.5-8.5 m & $\begin{array}{l}\text { Juniperus excelsa, Berberis } \\
\text { crataegina, Astragalus } \\
\text { angustifolius, } \\
\text { Geranium subcaulescens, } \\
\text { Myosotis sp., } \\
\text { Anthemis rosea Sm. }\end{array}$ & $\begin{array}{l}\text { Juniperus excelsa, Berberis } \\
\text { crataegina, Astragalus } \\
\text { angustifolius, Geranium } \\
\text { subcaulescens, Myosotis sp., } \\
\text { Anthemis rosea }\end{array}$ & $\begin{array}{l}\text { Juniperus excelsa, Berberis } \\
\text { crataegina, Alkanna areolata, } \\
\text { Ranunculus sp. }\end{array}$ & $\begin{array}{l}\text { Juniperus excelsa, Alkanna } \\
\text { areolata, Ranunculus sp. }\end{array}$ \\
\hline 8.5-9.5 m & $\begin{array}{l}\text { Juniperus excelsa, Berberis } \\
\text { crataegina, Astragalus sp., } \\
\text { Euphorbia sp., Taraxacum sp. }\end{array}$ & $\begin{array}{l}\text { Juniperus excelsa, Geranium } \\
\text { subcaulescens, Taraxacum sp., } \\
\text { Myosotis sp. }\end{array}$ & $\begin{array}{l}\text { Juniperus excelsa, Alkanna } \\
\text { areolata, Ranunculus sp., } \\
\text { Umbilicus erectus }\end{array}$ & $\begin{array}{l}\text { Poa bulbosa, } \\
\text { Alkanna areolata, Ranunculus } \\
\text { sp. }\end{array}$ \\
\hline 9.5-10.5 m & $\begin{array}{l}\text { Juniperus excelsa, Euphorbia sp., } \\
\text { Muscari sp. }\end{array}$ & $\begin{array}{l}\text { Berberis crataegina, Euphorbia } \\
\text { sp., Geranium subcaulescens, } \\
\text { Muscari sp. }\end{array}$ & $\begin{array}{l}\text { Berberis crataegina, Alkanna } \\
\text { areolata, Teucrium chamaedrys }\end{array}$ & $\begin{array}{l}\text { Juniperus excelsa, Alkanna } \\
\text { areolata, Capsella bursa-pastoris }\end{array}$ \\
\hline
\end{tabular}

odunsu bitkiler bulunmaktadır. J-2-B'deki tuzak çevresinde yaygin olarak bulunan otsu taksonlar Astragalus angustifolius Lam., Astragalus sp., Euphorbia sp., Geranium subcaulescens L'Hér. ex DC'dir. J-3-B'de ise, Alkanna areolata Boiss., Cicerbita sp., Euphorbia sp., Poa bulbosa L., ve Ranunculus sp. tuzağın en yakınında bulunan bitki taksonlarıdır (Tablo 2). J-4-B örnek alanı içerisinde yer alan polen tuzağı çevresinde odunsu bitkilerden sadece Juniperus excelsa bulunmaktadır. Polen tuzağı çevresinde yaygın olarak bulunan otsu bitki taksonları Cicerbita sp., Aubrieta pinardii Boiss., Alkanna areolata, Euphorbia sp., ve Ranunculus sp.'dir (Tablo 2).

\subsection{Tauber Polen Tuzaklarına Ait Polen Yoğunlukları}

Örnek alanlardaki (J-1-T, J-2-T, J-3-T ve J-4-T) polen tuzaklarının çevresinde bulunan bitkilerin 2015-2016 ve 20162017 yılları arasındaki yıllık polen yoğunlukları $\left(\mathrm{cm}^{2} / \mathrm{y} 1\right)$ belirlenmiştir (Şekil 2; Şekil 3). 
J-1-T örnek alanından elde edilen 2015-2016 y1lı y1llık polen yoğunluğu verilerine göre odunsu taksonlar içerisinde en fazla polen yoğunluğu $6726 \mathrm{~cm}^{2} /$ y1l ile Cedrus libani'ye aittir. Cedrus libani’yi $4624 \mathrm{~cm}^{2} / y 1$ polen yoğunluğu ile Pinus sp. ve 3936 $\mathrm{cm}^{2} / \mathrm{y} 1 \mathrm{l}$ polen yoğunluğu ile Juniperus sp. takip etmektedir (Şekil 2). Alandaki en fazla otsu bitki yoğunluğu $1070 \mathrm{~cm}^{2} / \mathrm{y} 1 \mathrm{l}$ ile Euphorbia'ya aittir (Şekil 3). J-2-T örnek alanından elde edilen yıllık polen yoğunluğu verilerine göre en fazla polen yoğunluğu $4576 \mathrm{~cm}^{2} /$ yıl ile yine Cedrus libani’ye aittir. Cedrus libani'yi $3290 \mathrm{~cm}^{2} / \mathrm{y}$ ll polen yoğunluğu ile Pinus sp. takip etmektedir. Alandaki diğer önemli odunsu bitki yıllık polen yoğunlukları $1765 \mathrm{~cm}^{2} /$ yıl ile Juniperus sp. ve $239 \mathrm{~cm}^{2} / \mathrm{y} 1 \mathrm{l}$ ile Quercus coccifera'ya aittir (Şekil 2). Alandaki en fazla otsu bitki polen yoğunluğunun ise $1555 \mathrm{~cm}^{2} / \mathrm{y} 1$ lile yine Euphorbia'ya ait olduğu görülmektedir (Şekil 3). J-3-T örnek alanından elde edilen yıllık polen yoğunluğu verilerine göre en fazla polen

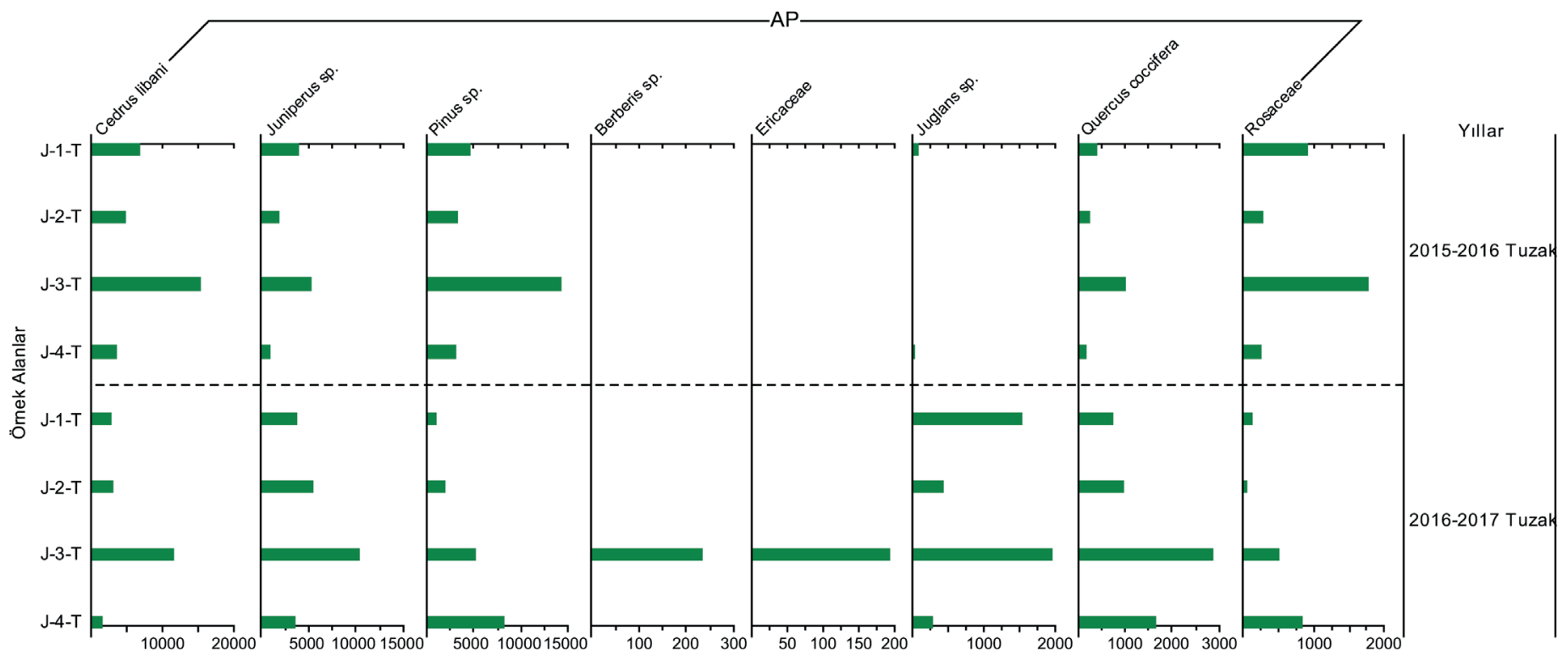

Şekil 2: Salurtepe Dağı Juniperus (J-T) ormanında 2015-2017 yılları arasında Tauber polen tuzaklarından elde edilen odunsu bitki (AP) polen yoğunlukları.

Figure 2: Pollen influxes of the arboreal plants (AP) obtained from Tauber pollen traps between 2015 and 2017 in the Juniperus (J-T) Forest of Salurtepe Mountain.

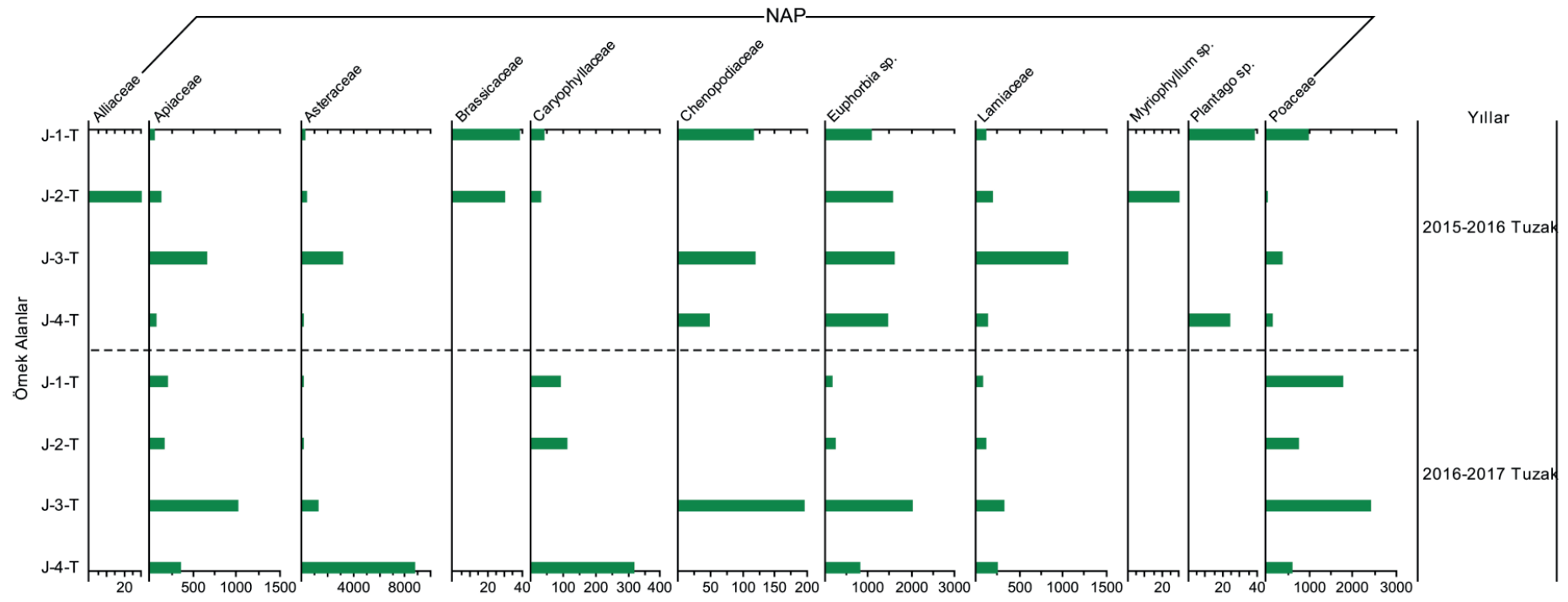

Şekil 3: Salurtepe Dağı Juniperus (J-T) ormanında 2015-2017 yılları arasında Tauber polen tuzaklarından elde edilen otsu bitki (NAP) polen yoğunlukları.

Figure 3: Pollen influxes of the non-arboreal plants (NAP) obtained from Tauber pollen traps between 2015 and 2017 in the Juniperus (J-T) Forest of Salurtepe Mountain . 
yoğunluğu $15212 \mathrm{~cm}^{2} / \mathrm{y} 1 \mathrm{l}$ ile Cedrus libani'ye aittir. Cedrus libani'yi $14210 \mathrm{~cm}^{2} / \mathrm{y} 1$ polen yoğunluğu ile Pinus sp. takip etmektedir. Alandaki diğer önemli odunsu bitki y1llık polen yoğunlukları $5189 \mathrm{~cm}^{2} /$ yıl ile Juniperus sp. ve $1769 \mathrm{~cm}^{2} / \mathrm{yll}$ ile Rosaceae familyasına aittir (Şekil 2). Otsu bitki polen yoğunluğu içerisinde özellikle Asteraceae familyasının yıllık polen yoğunluğu bu örnek alanda oldukça artmış ve $3125 \mathrm{~cm}^{2} / \mathrm{y} 11$ olarak tespit edilmiş̧ir (Şekil 3). J-4-T örnek alanından elde edilen yıllık polen yoğunluğu verilerine göre en fazla polen yoğunluğu $3527 \mathrm{~cm}^{2} / \mathrm{y} 1 \mathrm{l}$ ile Cedrus libani'ye aittir. Cedrus libani'yi $3000 \mathrm{~cm}^{2} / \mathrm{y} 11$ polen yoğunluğu ile yine Pinus sp. takip etmektedir. Alandaki diğer önemli odunsu bitki yıllık polen yoğunluğu $888 \mathrm{~cm}^{2} / \mathrm{y}$ ll ile Juniperus'a aittir (Şekil 2). Bu alandaki en önemli otsu bitki polen yoğunluğu J-1-T ve J-2-T'de olduğu gibi Euphorbia'ya $\left(1440 \mathrm{~cm}^{2} / \mathrm{y} 1\right.$ l) ait bulunmuştur (Şekil 3). Çalı̧̧ma alanındaki tüm örnek alanlar içerisinde toplam yıllık polen yoğunlukları 2015-2016 yılları için karşılaştırıldığında en fazla yıllık polen yoğunluğu $44281 \mathrm{~cm}^{2} / \mathrm{y} 1 \mathrm{l}$ ile J-3-T örnek alanında belirlenmiştir. En az toplam yıllık polen yoğunluğu ise $9767 \mathrm{~cm}^{2} /$ yıl ile J-4-T örnek alanındadır (Şekil 2; Şekil 3).

2016-2017 yılı y1llık polen yoğunluğu verilerine göre; J-1-T örnek alanında odunsu taksonlar içerisinde en fazla polen yoğunluğu $3628 \mathrm{~cm}^{2} / \mathrm{y}$ ll ile Juniperus sp.'ye ait çıkmıştır (Şekil 2). Juniperus sp.'yi $2642 \mathrm{~cm}^{2} / \mathrm{y} 1$ polen yoğunluğu ile Cedrus libani ve $1545 \mathrm{~cm}^{2} / \mathrm{y} 1$ polen yoğunluğu ile Juglans sp. takip etmektedir (Şekil 2). Alandaki en fazla otsu bitki polen yoğunluğu $1747 \mathrm{~cm}^{2} / \mathrm{y}$ ll ile Poaceae'ye aittir. Poaceae'yi 202 $\mathrm{cm}^{2} / \mathrm{y}$ ll polen yoğunluğu ile Apiaceae takip etmektedir (Şekil 3). J-2-T örnek alanından elde edilen yıllık polen yoğunluğu verilerine göre en fazla polen yoğunluğu $5333 \mathrm{~cm}^{2} / \mathrm{y} 1 \mathrm{l}$ ile yine Juniperus sp.'ye aittir. Juniperus sp.'yi $3007 \mathrm{~cm}^{2} / \mathrm{yll}$ polen yoğunluğu ile Cedrus libani ve $1929 \mathrm{~cm}^{2} / \mathrm{y} 1$ polen yoğunluğu ile Pinus sp. takip etmektedir (Şekil 2). Alandaki en fazla otsu bitki polen yoğunluğu $737 \mathrm{~cm}^{2} / \mathrm{y} 1 \mathrm{l}$ ile yine Poaceae'ye aittir. Poaceae'yi $227 \mathrm{~cm}^{2} / \mathrm{y} 1 \mathrm{l}$ polen yoğunluğu ile Euphorbia $\mathrm{sp}$. ve $170 \mathrm{~cm}^{2} / y 11$ polen yoğunluğu ile Apiaceae takip etmektedir (Şekil 3). J-3-T örnek alanından elde edilen y1llık polen yoğunluğu verilerine göre en fazla polen yoğunluğu $11449 \mathrm{~cm}^{2} /$ y1l ile Cedrus libani'ye aittir. Cedrus libani'yi $10211 \mathrm{~cm}^{2} / \mathrm{y}_{1} \mathrm{l}$ polen yoğunluğu ile Juniperus $\mathrm{sp} ., 5106 \mathrm{~cm}^{2} / \mathrm{y} 1$ polen yoğunluğu ile Pinus sp. ve $2862 \mathrm{~cm}^{2} / \mathrm{yll}$ ile Quercus coccifera takip etmektedir (Şekil 2). Alandaki en fazla otsu bitki polen yoğunluğu $2398 \mathrm{~cm}^{2} / \mathrm{y} 1 \mathrm{lile}$ Poaceae'ye aittir. Poaceae familyasını $2211 \mathrm{~cm}^{2} /$ yll polen yoğunluğu ile Euphorbia sp. ve $1238 \mathrm{~cm}^{2} / \mathrm{y} 1 \mathrm{l}$ polen yoğunluğu ile Asteraceae takip etmektedir (Şekil 3). J-4-T örnek alanından elde edilen yıllık polen yoğunluğu verilerine göre en fazla polen yoğunluğu $8037 \mathrm{~cm}^{2} / y 1$ ile Pinus'a aittir.
Pinus'u $3427 \mathrm{~cm}^{2} / \mathrm{y} 1$ polen yoğunluğu ile Juniperus ve 1655 $\mathrm{cm}^{2} / \mathrm{y} 11$ polen yoğunluğu ile Quercus coccifera ve $827 \mathrm{~cm}^{2} / \mathrm{y} 1$ polen yoğunluğu ile Rosaceae takip etmektedir (Şekil 2). Alandaki en fazla otsu bitki polen yoğunluğu $8746 \mathrm{~cm}^{2} / y 1 l$ ile Asteraceae'ye aittir. Asteraceae familyasını, $788 \mathrm{~cm}^{2} / \mathrm{y}_{11}$ polen yoğunluğu ile Euphorbia ve $591 \mathrm{~cm}^{2} / \mathrm{y} 11$ polen yoğunluğu ile Poaceae ve $355 \mathrm{~cm}^{2} / \mathrm{y} 1 \mathrm{l}$ polen yoğunluğu ile Apiaceae takip etmektedir (Şekil 3). Çalışma alanında tüm örnek alanlar içerisinde toplam y1llık polen yoğunlukları 2016-2017 y1lları için karşılaştırıldığında en fazla polen yoğunluğu $39685 \mathrm{~cm}^{2} / \mathrm{y} ı 1$ ile J-3-T örnek alanında belirlenmiştir. En az toplam yıllık polen yoğunluğu ise $12137 \mathrm{~cm}^{2} / \mathrm{y} 1 \mathrm{l}$ ile J-1-T örnek alanında belirlenmiştir (Şekil 2; Șekil 3).

\subsection{Kara Yosunu Örneklerine Ait Polen Yoğunlukları}

Çalışma alanı içerisinde tüm örnek alanlardaki (J-1-Y, J-2-Y, J-3-Y ve J-4-Y) polen tuzaklarının yakınından toplanan kara yosunu örneklerinden 2015-2016 ve 2016-2017 yılları arasında elde edilen yıllık odunsu ve otsu polen yoğunlukları $\left(\mathrm{cm}^{2} / \mathrm{y} 1 \mathrm{l}\right)$ hesaplanmıştır (Şekil 4; Şekil 5).

J-1-Y örnek alanından elde edilen 2015-2016 y1lı yıllık polen yoğunluğu verilerine göre en fazla polen yoğunluğu $786 \mathrm{~cm}^{2} / \mathrm{y} 1 \mathrm{l}$ ile Cedrus libani'ye ait çıkmış ve onu $491 \mathrm{~cm}^{2} / \mathrm{y} 1 \mathrm{l}$ polen yoğunluğu ile Pinus sp. takip etmiş̧ir (Şekil 4). Alandaki en fazla otsu bitki yoğunluğu $101 \mathrm{~cm}^{2} / y 1$ ile Poaceae familyasına aittir (Şekil 5). J-2-Y, J-3-Y ve J-4-Y örnek alanlarından elde edilen yıllık polen yoğunluğu verilerine göre en fazla polen yoğunluğu yine Cedrus libani ve Pinus sp.'de görülmüștür (Şekil 4). J-2-Y örnek alnında en fazla otsu bitki polen yoğunluğu $116 \mathrm{~cm}^{2} / \mathrm{y} 1 \mathrm{l}$ ile yine Poaceae familyasına ait çıkmışken, J-3-Y örnek alanında ise Asteraceae familyasının yıllık polen yoğunluğu yüksek bulunmuştur (Şekil 5). J-4-Y'de otsu bitki polen yoğunluğuna bakıldığında; bu alandaki en önemli otsu bitki taksonlar1 Lamiaceae $\left(189 \mathrm{~cm}^{2} / \mathrm{y} 1\right)$ ve Poaceae $\left(147 \mathrm{~cm}^{2} /\right.$ yıl) familyalarına ait çıkmıştır (Şekil 5).

Çalışma alanındaki tüm örnek alanlar içerisinde 2015-2016 yılı yıllık toplam polen yoğunlukları karşılaştırıldığında en fazla polen yoğunluğu $18853 \mathrm{~cm}^{2} / \mathrm{y}$ l lile J-3-Y örnek alanında tespit edilmiştir. En az toplam yıllık polen yoğunluğu ise $6502 \mathrm{~cm}^{2} / \mathrm{yl}$ ile J-1-Y örnek alanında belirlenmiştir (Şekil 4; Şekil 5).

J-1-Y örnek alanından elde edilen 2016-2017 y1lı yıllık polen yoğunluğu verilerine göre en fazla polen yoğunluğu $4585 \mathrm{~cm}^{2} / \mathrm{yll}$ ile Cedrus libani'ye aittir. Cedrus libani'yi $516 \mathrm{~cm}^{2} / \mathrm{y} 11$ polen yoğunluğu ile Juniperus ve $496 \mathrm{~cm}^{2} / \mathrm{y} 1 \mathrm{l}$ ile Pinus takip etmektedir 
(Şekil 4). Bu alandaki en fazla otsu bitki polen yoğunluğu $991 \mathrm{~cm}^{2} /$ yıl ile Apiaceae'ye aittir (Şekil 5). J-2-Y örnek alanından elde edilen yıllık polen yoğunluğu verilerine göre en fazla polen yoğunluğu $5150 \mathrm{~cm}^{2} / \mathrm{y} 1 \mathrm{l}$ ile yine Cedrus libani’ye ait bulunmuş ve onu sirasiyla Pinus (924 $\left.\mathrm{cm}^{2} / \mathrm{y} 1 \mathrm{l}\right)$, Juniperus $\left(532 \mathrm{~cm}^{2} / \mathrm{y} 1 \mathrm{l}\right) \mathrm{ve}$ Quercus coccifera (420 $\mathrm{cm}^{2} / \mathrm{y}$ ll) takip etmiştir (Şekil 4). Bu alandaki en fazla otsu bitki polen yoğunluğu $672 \mathrm{~cm}^{2} / \mathrm{y} 1 \mathrm{l}$ ile Poaceae familyasına aittir (Şekil 5). J-3-Y ve J-4-Y örnek alanlarından elde edilen yıllık polen yoğunluğu verilerine bakıldığında; en fazla polen yoğunlukları Cedrus libani, Pinus, Juniperus ve Quercus coccifera'ya ait çıkmıştır (Şekil 4). Bu örnek alanlardaki en fazla otsu bitki polen yoğunlukları incelendiğinde; J-3-Y'de $980 \mathrm{~cm}^{2} / y 1$ ile Brassicaceae familyası tespit edilirken, $\mathrm{J}-4-\mathrm{Y}^{\prime}$ de ise $304 \mathrm{~cm}^{2} / \mathrm{y}$ 1l Apiaceae familyası bulunmuştur (Şekil 5).

Çalışma alanındaki tüm örnek alanlar içerisinde 2016-2017 yılı yıllık toplam polen yoğunlukları karşılaştırıldığında en fazla polen yoğunluğu $9800 \mathrm{~cm}^{2} / \mathrm{y} 1 \mathrm{l}$ ile J-3-Y örnek alanında

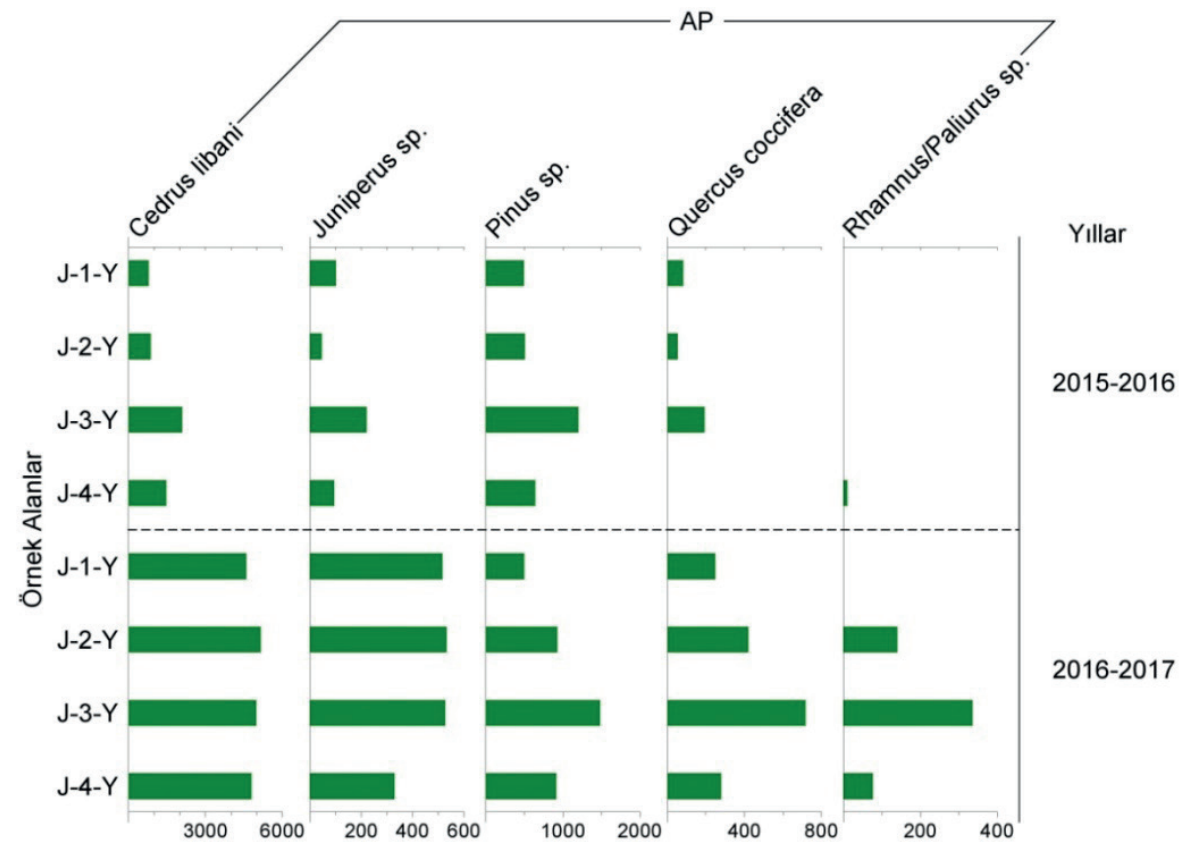

Şekil 4: Salurtepe Dağı-Elmalı Juniperus (J-Y) ormanında 2015-2017 yılları arasında kara yosunu örneklerinden elde edilen odunsu bitki taksonlarına ait polen yoğunlukları ( $\left.\mathrm{cm}^{2} / \mathrm{yıl}\right)$.

Figure 4: Pollen influxes of the arboreal plants (AP) obtained from moss samples between 2015 and 2017 in the Juniperus (J-T) Forest of Salurtepe Mountain.

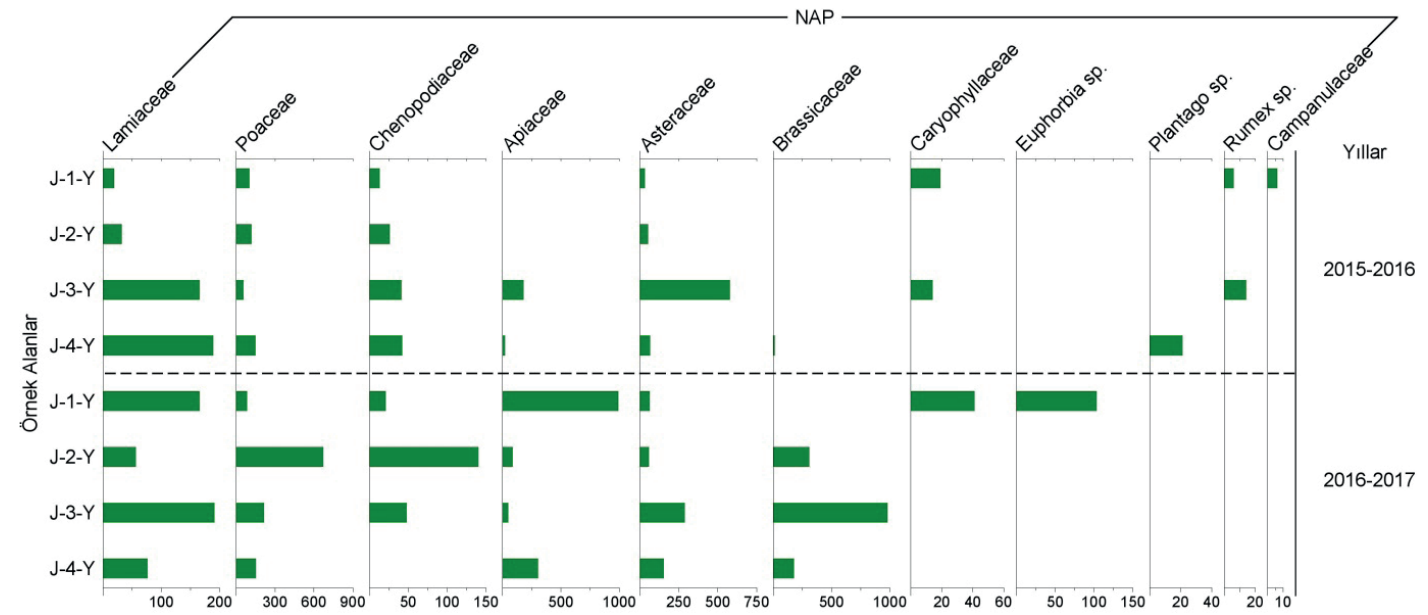

Şekil 5: Salurtepe Dağı Juniperus (J-Y) ormanında 2015-2017 yılları arasında kara yosunu örneklerinden elde edilen otsu bitki taksonlarına ait polen yoğunlukları ( $\left.\mathrm{cm}^{2} / \mathrm{yıl}\right)$.

Figure 5: Pollen influxes of the non-arboreal plants (NAP) obtained from moss samples between 2015 and 2017 in the Juniperus (J-T) Forest of Salurtepe Mountain . 
belirlenmiştir. En az toplam yıllık polen yoğunluğu ise $7243 \mathrm{~cm}^{2} /$ yıl ile J-4-Y örnek alanında tespit edilmiştir (Şekil 4; Şekil 5).

\subsection{Yüzey Sediman Örneğine Ait Polen Yoğunluğu}

Çalışma alanına yakın Elmalı Gölü’nden alınan yüzey sediman örneğine ait y1llık odunsu bitki polen yoğunluğu $10229 \mathrm{~cm}^{2} /$ y1l'dır. Bu polen yoğunluğunun içerisinde Pinus sp. $\left(9601 \mathrm{~cm}^{2} /\right.$ y1l) ilk sirada gelmekte ve onu Cedrus libani (329 $\mathrm{cm}^{2} / \mathrm{y} 1$ ) izlemektedir (Şekil 6). Polen yoğunluğuna sahip diğer önemli odunsu taksonlar arasında Olea europea, Quercus cerris type ve Quercus ilex type gelmektedir. Elmalı Gölü'nden alınan yüzey sediman örneğinden elde edilen yıllık otsu bitki polen yoğunluğu
$418 \mathrm{~cm}^{2} / y 1$ 'dır. Bu polen yoğunluğunun içerisinde Lactucaceae (239 $\left.\mathrm{cm}^{2} / \mathrm{y} 1 \mathrm{l}\right)$ ilk sirada gelmekte ve $90 \mathrm{~cm}^{2} / \mathrm{y} 1 \mathrm{l}$ ile Asteraceae ve Chenopodiaceae izlemektedir. Elmalı Gölü'nde sucul bitki polen yoğunluğu $59 \mathrm{~cm}^{2} /$ yıl ile Myriophyllum spicatum'a aittir (Şekil 6).

\section{TARTIŞMA}

\subsection{Tauber Polen Tuzaklarına ve Kara Yosunu Örneklerine Ait Polen Yoğunluklarının Karşılaştırılması}

Çalışma alanındaki Tauber polen tuzaklarından ve kara yosunu örneklerinden elde edilen 2015-2017 yıllarına ait toplam polen yoğunluğu karşılaştırılmıştır (Şekil 7). Bu karşılaştırmaya

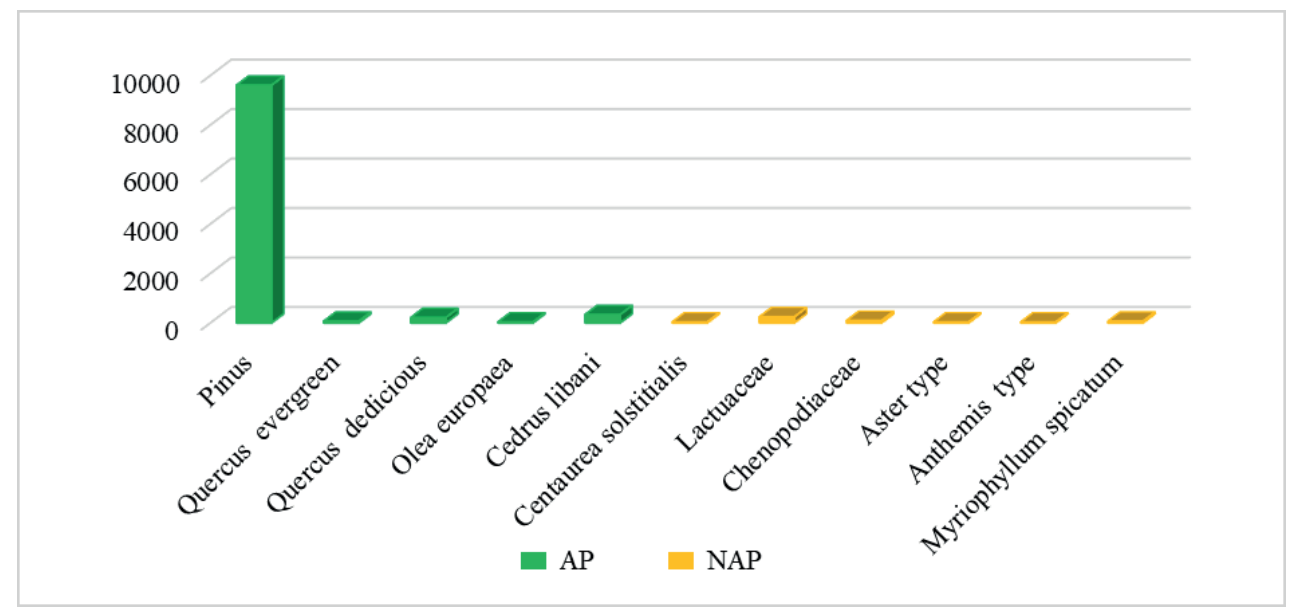

Şekil 6: Elmalı Gölü'nden elde edilen yüzey sediman örneğine ait AP ve NAP yoğunlukları. Figure 6: Arboreal and non-arboreal pollen influxes obtained from surface sample of Elmalı Lake.

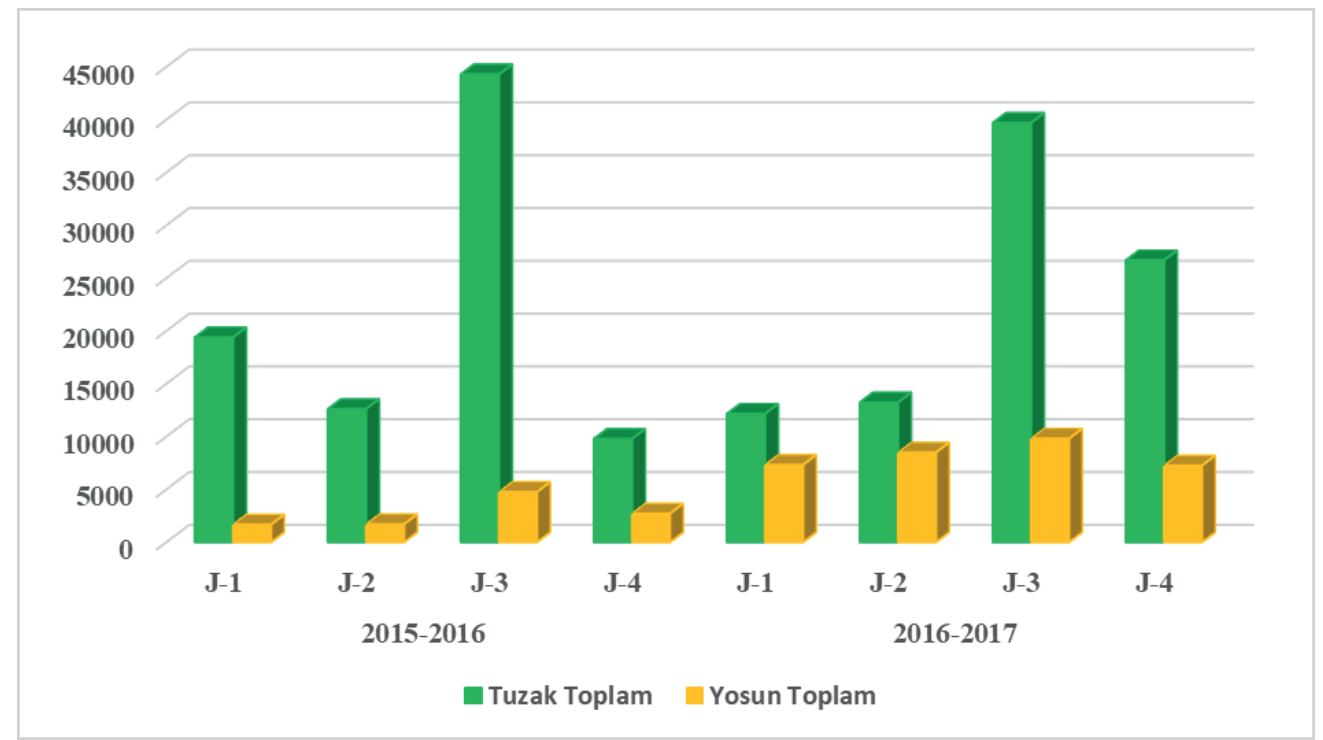

Şekil 7: Salurtepe Dağı Juniperus (J) ormanındaki tüm örnek alanlara ait 2015-2017 yılları arası tuzak ve kara yosunu örneklerinin toplam polen yoğunlukları ( $\left.\mathrm{cm}^{2} / \mathrm{yll}\right)$.

Figure 7: Total pollen influxes of Tauber pollen traps and moss samples in the all sample areas of Juniperus Forest of Salurtepe Mountain between 2015 and 2017. 


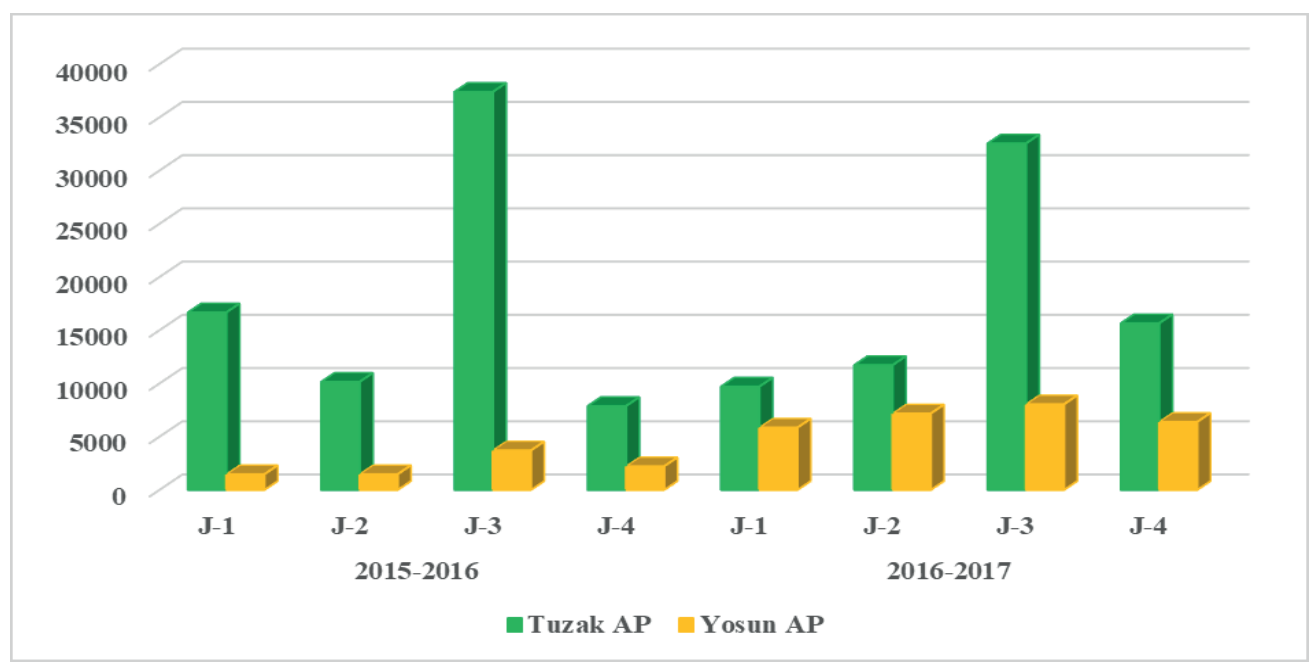

Şekil 8: Salurtepe Dağı Juniperus (J) ormanındaki tüm örnek alanlara ait 2015-2017 yılları arası tuzak ve kara yosunu örneklerinin toplam AP yoğunlukları $\left(\mathrm{cm}^{2} / \mathrm{yll}\right)$.

Figure 8: Total arboreal pollen influxes of Tauber pollen traps and moss samples in the all sample areas of Juniperus Forest of Salurtepe Mountain between 2015 and 2017.

göre 2015-2017 yılları arasında tuzaklardan elde edilen toplam polen yoğunluklarının tüm örnek alanlarda kara yosunu örneklerinden elde edilen toplam polen yoğunluklarından daha fazla olduğu görülmektedir. 2015-2016 yılında tuzaklardan elde edilen polen yoğunluğu J-1 ve J-2 no'lu örnek alanlarda azalış gösterirken, 2016-2017 yılında artı̧̧ göstermektedir. Tüm örnek alanlarda tuzaklardan elde edilen toplam polen yoğunluklarına bakıldığında en fazla polen yoğunluğunun 2015-2017 yıllarında J-3 no'lu örnek alanda olduğu görülmektedir (Şekil 7). 20152016 yılında kara yosunu örneklerinden elde edilen toplam polen yoğunluğu J-1 ve J-2 no'lu örnek alanlarda hemen hemen aynı değeri gösterirken, 2016-2017 yılında artış göstermektedir. Tüm örnek alanlardaki kara yosunu örneklerinden elde edilen toplam polen yoğunluklarına bakıldığında en fazla polen yoğunluğu 2015-2016 ve 2016-2017 yılında J-3 no'lu örnek alanda görülmektedir. 2015-2017 yıllarına ait tuzak ve kara yosunu örneklerinden elde edilen toplam AP yoğunluğu ve toplam AP oranları genel olarak tuzaklara ait polen verilerinde daha yüksektir (Şekil 8).

\subsection{Tauber Polen Tuzaklarına ve Kara Yosunu Örneklerine Ait Yıllık Polen Yoğunluğu Verilerinin Güncel Bitki Türleri ile Karşılaştırılması}

Çalışma alanında hem polen tuzaklarında hem de kara yosunu örneklerinde (2015-2016 ve 2016-2017) yapılan polen analiz sonuçlarına göre yıllık polen yoğunluğu $\left(\mathrm{cm}^{2} / \mathrm{yl}\right)$ en fazla olan odunsu taksonları Cedrus libani, Pinus sp., Juniperus, Quercus coccifera, Rosaceae, Juglans, Pinus oluştururken; yıllık polen yoğunluğu en fazla otsu taksonları ise Euphorbia, Asteraceae,
Poaceae, Apiaceae, Rosaceae, Lamiaceae, Caryophyllaceae, Brassicaceae ve Chenopodiaceae oluşturmaktadır. Tuzakların etrafındaki ilk $7.5 \mathrm{~m}$ ve üzeri mesafede bitki örtüsü incelediğinde; yukarıda belirtilen en fazla polen yoğunluğuna sahip taksonların güncel vejetasyonu yansıttığı görülmektedir.

Çalışma alanındaki tuzaklara ait polen yoğunlukları karşılaştırıldığında 2015-2016 yılında toplam polen yoğunlukları J-1-T ve J-3-T no'lu örnek alanlarda daha fazladır. J-2-T örnek alanında her iki yıl da birbirine yakın seyretmişken J-4-T örnek alanında 2016-2017 yılında önemli bir artış görülmektedir. Kara yosunu örneklerinde 2016-2017 yılları arasında tüm örnek alanlardaki yıllık toplam polen yoğunlukları 2015-2016 yılına göre artış göstermiştir (Şekil 8).

\section{SONUÇ}

Salurtepe Dağı Juniperus (J) ormanında güncel polen dağılımının belirlenmesi için birim toprak alanına düşen polen miktarı yıllık olarak hesaplanmıştır. Toprağa düşen polen yoğunluk verileri tuzakların bulunduğu örnek alanlara göre farkl111klar göstermiştir. Bu farklılıklar AP ve NAP yoğunlukları bakımından karşılaştırıldığında genel olarak en fazla yoğunluk odunsu bitki taksonlarına (AP) aittir. Bu sonuç, birim toprak alanına düşen polen miktarının büyük oranda belirlenen örnek alanlardaki baskın taksonlara ait olduğunu göstermektedir.

Tauber polen tuzakları ve kara yosunu örneklerinin yıllık toplam polen yoğunluğu $\left(\mathrm{cm}^{2} / \mathrm{y} 1 \mathrm{l}\right)$ karşılaştırıldığında en fazla polen yoğunluğu tuzaklardan elde edilmiştir. Bu çalışmadan elde 
edilen güncel polen yoğunluk verilerine göre, Karlığlu (2011), Karlığlu ve Akkemik (2015); Karlığlu vd. (2014; 2015) ve Şenkul vd. (2018c) çalışmalarındaki gibi Tauber polen tuzakları içerisinde bulundukları vejetasyonu yansıtmaktadır.

Hem polen tuzakları hem de yosun örneklerine ait yıllık polen yoğunluğu verilerine bakıldığında, 2015-2017 yılları arasında görülen otsu ve odunsu taksonlar vejetasyon halkası içinde de bulunmaktadır. Ancak, odunsu taksonlardan Pinus'un vejetasyon halkası içinde bulunmamasına rağmen polen yoğunluğunun yüksek olmasının nedeni polenlerinin sahaya rüzgarla çok uzak mesafelerden taşınmasıdır.

Finansal Destek: Bu çalışma TÜBITTAK 3501 programı kapsamında 214O249 numaralı "Teke Yarımadası Ormanlarında Güncel Polen Dağıllımının ve Mikro İklim Koşullarının Belirlenmesi” adlı proje (Şenkul vd., 2018a) tarafindan desteklenmiştir.

Teșekkür: Güncel bitki örneklerinin toplanması ve teşhisindeki yardımlarından dolayı Afyon Kocatepe Üniversitesi Fen Edebiyat Fakültesi Moleküler Biyoloji ve Genetik Bölümü öğretim üyesi Prof. Dr. Mustafa Kargıoğlu'na teşekkürlerimizi sunarız.

\section{KAYNAKLAR}

Aytuğ, B. (1967). Polen Morfolojisi ve Türkiye'nin Önemli Gymnospermleri Üzerinde Palinolojik Araşttrmalar. Kutulmuş Matbaası, İstanbul.

Aytuğ, B., Aykut, S., Merev, N. Ve Edis, G. (1971). İstanbul çevresi bitkilerinin polen atlası. İ.Ü. Orman Fakültesi Yayınları No:174, Kutulmuş Matbaası, İstanbul.

Bakker, J., Kaniewski, D., Verstraeten, G., Laet, V.D., \& Waelkens, M. (2011). Numerically derived evidence for late-Holocene climate change and its impact on human presence in the southwest Taurus Mountains, Turkey. The Holocene, 22, 425-438.

Bottema, S., Woldring, H. (1984). Late quaternary vegetation and climate of southwestern Turkey Part II. Palaeohistoria, 26, 245249.

Doğan, M. (2017). Fosil ve Güncel Polen Analizleri Işlğında Mucur Çevresinin Geç Holosen Paleovejetasyonu. (Yüksek Lisans Tezi). Süleyman Demirel Üniversitesi, Sosyal Bilimler Enstitüsü, Coğrafya Anabilim Dalı, Isparta.

Eastwood, W.J. (1997). The Palaeoecological Record of Holocene Environmental Change in Southwest Turkey (Doctoral dissertation). University of Wales.

Erdtman, G. (1952). Pollen Morphology and Plant TaxonomyAngiosperms. The Chronica Botanica Company, Waltham, Mass., U.S.A.
Erdtman, G. (1957). Pollen and Spore Morphology / Plant Taxonomy Gymnospermae, Pteridopyhta, Bryophyta. Stockholm.

Faegri, K., Iversen, J. (1975). Textbook of Pollen Analysis, 3rd ed., New York, Hafner.

Filipova-Marinova, M.V., Kvavadze, E., Connor, S., Sjögren, P. (2010). Estimating absolute pollen productivity for some European Tertiaryrelict taxa. Vegetation History and Archaeobotany, 19 (4), 351-364.

Gerasimidis, A., Panajiotidis, S., Hicks, S., Athanasiadis, N. (2006). An eight-year record of pollen deposition in the Pieria Mountains (N Greece) and its significance for interpreting fossil pollen assemblages. Review of Palaeobotany and Palynology, 141, 231-243.

Giesecke, T., Fontana, S.L., van der Knaap, W.O., Pardoe, H.S., Pidek, I.A. (2010). From early pollen trapping experiments to the Pollen Monitoring Programme. Vegetation History and Archaeobotany, 19 (4), 247-258.

Glew, J.R. (1995). Conversion of shallow water gravity coring equipment for deep water Operation, Journal of Paleolimnology, $14,83-88$.

Hesse, M., Zetter, R., Halbritter, H., Weber, M., Buchner, R., FroschRadivo, A., Ulrich, S. (2009). Pollen Terminology an illustrated handbook. Austria, Springer Wien New York.

Hicks, S. (1985). Modern pollen deposition records from Kuusamo, Finland. 1. Seasonal and annual variation. Grana, 24, 167-184.

Hicks, S., Hyvärinen, V.P. (1986). Sampling modern pollen deposition by means of "Tauber traps": some considerations. Pollen et Spores, 28, 219-242.

Hicks, S., Ammann, B., Latalowa, M., Pardoe, H., Tinsley, H. (1996). European Pollen Monitoring Programme: Project Description and Guidelines. University of Oulu, $28 \mathrm{pp}$.

Hicks, S., Tinsley, H., Huusko, A., Jensen, C., Hattestrand, M., Gerasimides, A., Kvavadze, E. (2001). Some comments on spatial variation in arboreal pollen deposition: First records from the Pollen Monitoring Programme (PMP). Review of Palaeobotany and Palynology, 117, 183-194.

Iwanami, Y., Sasakuma, T., Yamada, Y. (1988). Pollen: Illustrations and Scanning Electronmicrographs. Kodonsha-Tokyo.

Karlıoğlu, N. (2011). Istranca ve Belgrad Ormanlarında Güncel Polen Dağllımının İncelenmesi. (Doktora Tezi). İstanbul Üniversitesi Fen Bilimleri Enstitüsü, İstanbul.

Karlıoğlu, N. ve Akkemik, Ü. (2012). İ.Ü. Orman Fakültesi Araştırma Ormanı'nda Eylül 2007-Ağustos 2009 Dönemi Güncel Polen Dağllımı, Journal of the Faculty of Forestry, Istanbul University, 62 (2), 145-158

Karlığlu, N., Caner, H., Akkemik, Ü. (2014). Modern pollen distribution at Iğneada waterlogged forests between the periods September 2007-August 2009, Eurasian Journal of Forest Science, 2 (2), 7-17.

Karlıoglu, N., Caner, H., Akkemik, Ü., Köse, N., Kındap, T. (2015). Modern Pollen Monitoring of Native Trees in Belgrad Forest, Istanbul (Northwestern Turkey), Comptes rendus de I'Académie bulgare de Sciences, 68 (1), 39-48. 
Kaniewski, D., De Laet, V., Paulissen, E., Waelkens, M. (2007). Longterm effects of human impact on mountainous ecosystems, western Taurus Mountains, Turkey. Journal of Biogeography, 1-23.

Kvavadze, E. (1999). The first results of the pollen monitoring programme in the Caucasus Mountains (Georgia). Acta Palaeobotanica, 39, 171-177.

Moore, P.D., Webb, J.A., Collinson, M.E. (1991). Pollen Analysis. Blackwell, Oxford.

Pidek, I.A. (2007). Nine-year record of Alnus pollen deposition in the Roztocze region (SE Poland) with relation to vegetation data. Acta Agrobotanica, 60, 57-64.

Şenkul, Ç., Doğan, M. (2018). Fosil ve güncel polen analizleri ışığında Mucur Obruk Gölü çevresinin Paleovejetasyon değişimleri. Türk Coğrafya Dergisi, 70, 19-28. DOI:19.17211/tcd.342955.

Şenkul, Ç., Karlığlu Kılıç, N., Kargığlu, M., Eastwood, J.W. (2018a.). Teke Yarımadası Ormanlarında Güncel Polen Dağılımının ve Mikro İklim Koşullarının Belirlenmesi, TÜBİTAK Proje No: 214O249, Isparta.

Şenkul, Ç., Karlıŏ̆lu Kılıç, N., Kargığ̆lu, M., Kulakoğlu, F., Eastwood, J.W., Doğan, U., (2018b.) Kültepe (Kayseri) Çevresinin Fosil ve Güncel Polen Analizleri Işığında Holosen Ortamsal Değişimi, TÜBİTAK Proje No: 114Y578, Isparta.

Şenkul, Ç., Karlığlu Kılıç, N., Doğan, M., Eastwood, W.J. (2018c). Modern pollen distribution of the Teke Peninsula forests: The case of the Ördübek Highland, Eurasian Journal of Forest Science, 6(4): $58-75$.
Stockmarr, J. (1971). Tablets with spores used in absolute pollen analysis. Pollen et Spores, 13, 615-621.

Tauber, H. (1974). A static non-overload pollen collector. New Phytologist, 73, 359-369.

Tonkov, S., Hicks, S., Bozilova, E., Atanassova, J. (2001). Pollen monitoring in the Central Rila Mts., Southwestern Bulgaria: case studies from pollen traps, surface samples for the period 1994 1999. Review of Palaeobotany and Palynology, 117, 167-182.

van der Knaap, W.O., van Leeuwen, J.F.N., Ammann, B. (2001). Seven years of annual pollen influx at the forest limit in the Swiss Alps studied by pollen traps: relations to vegetation and climate. Review of Palaeobotany and Palynology, 117, 31-52.

van Zeist, W., Woldring, H., Stapert, D. (1975). Late quaternary vegetation and climate of southwestern Turkey. Palaeohistoria, 17, 55-143.

Vermoere, M., Bottema, S., Vanhecke, L., Waelkens, M., Paulissen, E., Smets, E. (2002). Palynological evidence for late-Holocene human occupation recorded in two wetlands in SW Turkey. The Holocene, 12, 569-584.

Wodehouse, R. P. (1935). Pollen Grains. Hafner Publishing Company, New York, 574 pp. 